\title{
ON THE $L^{2}$ BOUNDEDNESS THEOREM OF NON-HOMOGENEOUS FOURIER INTEGRAL OPERATORS IN $R^{n}$
}

\author{
By Kenji ASADA
}

\section{$\S 1$. Introduction and Notations.}

A Fourier integral operator is an integral transformation of the form

$$
A f(x)=(2 \pi)^{-n} \int_{R^{n}} e^{\imath S(x, \xi)} a(x, \xi) \hat{f}(\xi) d \xi,
$$

where

$$
\hat{f}(\xi)=\int_{R^{n}} e^{-\imath y \cdot \xi} f(y) d y
$$

is the Fourier transform of $f$ defined on $\boldsymbol{R}^{n}$. We call $S(x, \xi)$ its phase function and $a(x, \xi)$ its symbol function (cf. Hörmander [8]). When $S(x, \xi)=x \cdot \xi$, a Fourier integral operator becomes a pseudo-differential operator.

If a symbol function satisfies the inequalities

$$
\left|\partial_{x}^{\alpha} \partial_{\xi}^{\beta} a(x, \xi)\right| \leqq C_{\alpha, \beta}(1+|\xi|)^{\delta|\alpha|-\rho|\beta|}
$$

$(0 \leqq \delta \leqq \rho \leqq 1, \delta<1)$, Calderón-Vaillancourt [5] proved that the pseudo-differential operator with symbol $a(x, \xi)$ is $L^{2}$ bounded, and Fujiwara [7] and Kumano-go [12] proved the $L^{2}$ boundedness theorem of the Fourier integral operator.

If we take $\lambda(\xi)=(1+|\xi|)^{(\rho+\delta) / 2}$, then such a symbol function $a(x, \xi)$ satisfies the inequalities

$$
\left|\partial_{x}^{\alpha} \partial_{\xi}^{\beta} a(x, \xi)\right| \leqq C_{\alpha, \beta} \lambda(\xi)^{|\alpha|-|\beta|} .
$$

In this paper we consider the case that a weight function $\lambda(\xi)$ is more general in $\xi$ (See Definition 1 in Section 2), and we shall prove the $L^{2}$ boundedness theorem of the Fourier integral operator $A$ with symbol function $a(x, \xi)$ satisfying the inequalities (3).

We use the standard notations for functions and operators. A multi-index is a sequence $\alpha=\left(\alpha_{1}, \cdots, \alpha_{n}\right)$ of non-negative integers (the number $n$ will usually be clear from the context). If $\alpha$ is a multi-index and $x=\left(x_{1}, \cdots, x_{n}\right), \xi=$ $\left(\xi_{1}, \cdots, \xi_{n}\right)$ in $\boldsymbol{R}^{n}$, we set

Received September 20, 1983 


$$
\begin{aligned}
& |\alpha|=\alpha_{1}+\cdots+\alpha_{n}, \quad \alpha !=\alpha_{1} ! \cdots \alpha_{n} !, \\
& x \cdot \xi=x_{1} \xi_{1}+\cdots+x_{n} \xi_{n}, \quad|x|=\left(x_{1}^{2}+\cdots+x_{n}^{2}\right)^{1 / 2}, \\
& \langle\xi\rangle=\left(1+|\xi|^{2}\right)^{1 / 2}, \quad x^{\alpha}=x_{1}^{\alpha_{1}} \cdots x_{n}^{\alpha_{n}}, \\
& \partial_{x}^{\alpha}=\partial_{x_{1}}^{\alpha_{1}} \cdots \partial_{x_{n}}^{\alpha_{n}}, \quad \partial_{x_{\jmath}}=\partial / \partial_{c_{n}}, \quad \jmath=1, \cdots, n .
\end{aligned}
$$

If $f=f(x)$ is a function of $x$, then we set

$$
\begin{aligned}
& \nabla_{x} f=\left(\partial_{x_{1}} f, \cdots, \partial_{x_{n}} f\right), \\
& \|f\|=\left(\int_{\boldsymbol{R}^{n}}|f(x)|^{2} d x\right)^{1 / 2} .
\end{aligned}
$$

For a positive number $r$, we denote by $\chi_{r}$ the characteristic function of the ball $\{x ;|x| \leqq r\}$. We denote by $\mathcal{S}\left(\boldsymbol{R}^{n}\right)$ the Schwartz space of rapidly decreasing functions on $\boldsymbol{R}^{n}$, and by $L^{2}\left(\boldsymbol{R}^{n}\right)$ the set of measurable functions on $\boldsymbol{R}^{n}$ such that $\|f\|$ is finite. If $A$ is an operator, we denote the operator norm of $A$ in $L^{2}\left(\boldsymbol{R}^{n}\right)$ by $\|A\|$.

We adopt the following convention on constants: unless otherwise stated, constants $C, C^{\prime}$ vary from statement to statement, but depend only on the constants previously chosen.

\section{$\S 2$. Definitions and Results.}

Definition 1. We say that a $C^{\infty}$ real valued function $\lambda_{1}(\xi)$ defined on $\boldsymbol{R}^{n}$ is a basic weight function if $\lambda_{1}(\xi)$ satisfies the following conditions:

(W-1) There exist a positive constant $C_{1}$ such that

for all $\xi$ in $\boldsymbol{R}^{n}$.

$$
1 \leqq \lambda_{1}(\xi) \leqq C_{1}\langle\xi\rangle
$$

(W-2) For any multi-index $\alpha$ there exists a positive constant $C_{a}$ such that

for all $\xi$ in $\boldsymbol{R}^{n}$.

$$
\left|\partial_{\xi}^{\alpha} \lambda_{1}(\xi)\right| \leqq C_{\alpha} \lambda_{1}(\xi)^{1-|\alpha|}
$$

And we set $\lambda_{\varepsilon}(\xi)=\lambda_{1}(\xi)^{\varepsilon}$, where $0 \leqq \varepsilon \leqq 1$. Then we say that $\lambda_{\varepsilon}(\xi)$ is a weight function of type $\varepsilon$ induced from $\lambda_{1}(\xi)$.

Remark 1. The weight function of the above type is used in Boutet de Monvel [4] and Kumano-go [10]. Beals-Fefferman [2], Beals [3] and Kumanogo-Taniguchi [11] define more general weight functions also depending on the $x$-variables in order to develop the calculus of pseudo-differential operators. In the context of such general weight functions we would be able to consider the $L^{2}$ boundedness of Fourier integral operators. In [13] we have attempted some generalizations.

DEFINITION 2. Let $\lambda(\xi)$ be a weight function of type $\varepsilon(0 \leqq \varepsilon \leqq 1)$ and $\mu$ a 
real number. We say that a $C^{\infty}$ function $a(x, \xi)$ defined on $\boldsymbol{R}^{n} \times \boldsymbol{R}^{n}$ is a symbol function of order $\mu$ if $a(x, \xi)$ satisfies the condition:

$(\mathrm{S}-\mu)$ For any two multi-indices $\alpha$ and $\beta$ there exists a constant $C_{\alpha, \beta}$ such that for all $(x, \xi)$ in $\boldsymbol{R}^{n} \times \boldsymbol{R}^{n}$ the estimate

holds.

$$
\left|\partial_{x}^{\alpha} \partial_{\xi}^{\beta} a(x, \xi)\right| \leqq C_{\alpha, \beta} \lambda(\xi)^{\mu+|\alpha|-|\beta|}
$$

Remark 2. The set $S^{\mu}(\lambda)$ of all symbol functions of order $\mu$ corresponding to a weight function $\lambda(\xi)$ is a Fréchet space with semi-norms $|\cdot|_{\mu, k}$, where

$$
|a|_{\mu, k}=\sum_{|\alpha|+|\beta| \xi k} \sup _{(x, \xi) \in \boldsymbol{R}^{n} \times \boldsymbol{R}^{n}} \frac{\left|\partial_{x}^{\alpha} \partial_{\xi}^{\beta} a(x, \xi)\right|}{\lambda(\xi)^{\mu+1 \alpha|-1 \beta|}}
$$

for any non-negative integer $k$.

EXAMPLE 1. $\lambda_{1}(\xi)=\langle\xi\rangle$ is a basic weight function and $\lambda_{\tau}(\xi)=\langle\xi\rangle^{\tau}$ is a weight function of type $\tau(0 \leqq \tau<1)$. Then the symbol class $S^{\mu}\left(\lambda_{\tau}\right)$ is $S_{\tau, \tau}^{\tau \mu}$ in Hörmander [8]. And $S_{\rho, \delta}^{\mu} \subset S^{\nu}\left(\lambda_{\tau}\right)$ if $\delta \leqq \tau \leqq \rho, \tau<1$ and $\nu \geqq \tau \mu$.

EXAMPLE 2. We set

$$
\lambda_{1}(\xi)=\left(1+\sum_{\jmath=1}^{n} \xi_{\jmath}^{2 m_{\jmath}}\right)^{1 / 2 m}, \quad m=\max _{1 \leqq j \leqq n}\left\{m_{\jmath}\right\} .
$$

Then $\lambda_{1}(\xi)$ is a basic weight function.

Definition 3. We say that a $C^{\infty}$ real valued function $S(x, \xi)$ defined on $\boldsymbol{R}^{n} \times \boldsymbol{R}^{n}$ is a phase function if $S(x, \xi)$ satisfies the following conditions :

(P-1) For any two-multi-indices $\alpha$ and $\beta$ such that $|\alpha|+|\beta| \geqq 2$ there exists a constant $C_{\alpha, \beta}$ such that

$$
\left|\partial_{x}^{\alpha} \partial_{\xi}^{\beta} S(x, \xi)\right| \leqq C_{\alpha, \beta} \lambda(\xi)^{|\alpha|-|\beta|}
$$

for any $(x, \xi)$ in $\boldsymbol{R}^{n} \times \boldsymbol{R}^{n}$.

(P-2) There exists a positive constant $\delta_{0}>0$ such that

$$
\inf _{(x, \xi) \in R^{n} \times R^{n}}\left|\operatorname{det}\left(\partial_{x} \partial_{\xi_{k}} S(x, \xi)\right)\right| \geqq \delta_{0} .
$$

DEFINITION 4. Let $\lambda(\xi)$ be a weight function of type $\varepsilon(0 \leqq \varepsilon<1)$. Corre.. sponding to $a(x, \xi)$ in $S^{\mu}(\lambda)$ and a phase function $S(x, \xi)$ we define a Fourier integral operator $A$ on smooth functions by the formula

$$
A u(x)=\int_{R^{n}} e^{i S(x, \xi)} a(x, \xi) \hat{u}(\xi) d \xi,
$$

and $d \xi=(2 \pi)^{-n} d \xi$.

The defining integral in the right-hand side of (5) converges absolutely at least for any function $u$ in $\mathcal{S}\left(\boldsymbol{R}^{n}\right)$. For we have the estimate 


$$
\left|e^{\imath S(x, \xi)} a(x, \xi) \hat{u}(\xi)\right| \leqq C_{N}\langle\xi\rangle^{\varepsilon \mu-N},
$$

where $N$ is any positive integer.

Our result is :

THEOREM 1. Let $\lambda(\xi)$ be a werght function of type $\varepsilon(0 \leqq \varepsilon<1)$. Suppose that a symbol function $a(x, \xi)$ is in $S^{0}(\lambda)$ and a phase function $S(x, \xi)$ satisfies (P-1) and (P-2). Then the Fourier integral operator $A$ is $L^{2}$ bounded and has the estimate that

$$
\|A u\| \leqq C_{m}|a|_{0, m}\|u\|,
$$

where $m$ is an integer such that $m>2 n /(1-\varepsilon)$.

EXAMPLE 3. If $\lambda(\xi)=1$, then the Fourier integral operator turns out to be an oscillatory integral transformation in Fujiwara [6] and Asada-Fujiwara [1].

EXAMPLE 4. Fujiwara [7] and Kumano-go [12] proved the $L^{2}$ boundedness theorem of Fourier integral operators with symbol functions in $S_{\rho, \delta}^{0}(0 \leqq \delta \leqq \rho \leqq 1$, $\delta<1)$, under the condition that $S(x, \xi)$ satisfies $(\mathrm{P}-3)$, not $(\mathrm{P}-1)$.

(P-3) For any two multi-indices $\alpha$ and $\beta$ with $|\alpha|+|\beta| \geqq 2$, there exists a constant $C_{\alpha, \beta}$ such that

$$
\left|\partial_{x}^{\alpha} \partial_{\xi}^{\beta} S(x, \xi)\right| \leqq C_{\alpha, \beta}\langle\xi\rangle^{1-|\beta|} .
$$

Applying Theorem 1 to this case we have the following

COROLLARY. Let $\tau$ be a real number such that $0 \leqq \tau<1$. We assume the following conditions:

(i) For any multi-indices $\alpha$ and $\beta$ there exists a constant $C_{\alpha, \beta}$ such that

$$
\left|\partial_{x}^{\alpha} \partial_{\xi}^{\beta} a(x, \xi)\right| \leqq C_{\alpha, \beta}\langle\xi\rangle^{\tau(|\alpha|-|\beta|)} .
$$

(ii) There exists a positive constant $\delta_{0}$ such that

$$
\left|\operatorname{det}\left(\partial_{x_{j}} \partial_{\xi_{k}} S(x, \xi)\right)\right| \geqq \delta_{0} .
$$

(iii) For any multi-indices $\alpha$ and $\beta$ with $|\alpha|+|\beta| \geqq 2$ there exasts a constant $C_{\alpha, \beta}$ such that

$$
\left|\partial_{x}^{\alpha} \partial_{\xi}^{\beta} S(x, \xi)\right| \leqq C_{\alpha, \beta}\langle\xi\rangle^{\tau(|\alpha|-|\beta|)} .
$$

Then the Fourier integral operator $A$ is $L^{2}$ bounded and has the estrmate that

$$
\|A u\| \leqq C|a|_{0, m}\|u\|,
$$

where $m$ is an integer such that $m>2 n /(1-\tau)$.

Remark 3. By Plancherel's theorem we have only to prove that the integral operator 


$$
f(\xi) \rightarrow \int_{R^{n}} e^{i S(x, \xi)} a(x, \xi) f(\xi) d \xi
$$

is $L^{2}$ bounded. We again denote by $A$ this integral operator.

\section{$\S 3$. Proof of Theorem.}

LEMMA 1. Let $\lambda_{1}(\xi)$ be a basic werght function and $\lambda_{\varepsilon}(\xi)=\lambda_{1}(\xi)^{s} \quad(0 \leqq \varepsilon<1)$. Then $\lambda_{\varepsilon}(\xi)$ satisfies the following estimates:

$$
1 \leqq \lambda_{\varepsilon}(\xi) \leqq C_{\varepsilon}\langle\xi\rangle^{\varepsilon} .
$$

(W-4) For any multz-index $\alpha$ there exists a constant $C_{\varepsilon, \alpha}$ such that

$$
\left|\partial_{\xi}^{\alpha} \lambda_{\varepsilon}(\xi)\right| \leqq C_{\varepsilon, \alpha} \lambda_{\varepsilon}(\xi) \lambda_{1}(\xi)^{-|\alpha|} .
$$

This lemma is an immediate consequence of Definition 1. So we omit its proof.

Lemma 2. Let $\lambda_{1}(\xi)$ be a basic werght function. Then there exist positive constants $r_{0}$ and $C$ such that $C^{-1} \leqq \lambda_{1}(\xi) / \lambda_{1}(\eta) \leqq C$ whenever $|\xi-\eta| \leqq r_{0} \lambda_{1}(\xi)$.

Proof. We note from (W-2) that for $|\alpha|=1$

$$
\left|\partial_{\xi}^{\alpha} \lambda_{1}(\xi)\right| \leqq C \text {. }
$$

By the mean value theorem we have

$$
\left|\lambda_{1}(\eta)-\lambda_{1}(\xi)\right| \leqq C|\eta-\xi| \leqq C r_{0} \lambda_{1}(\xi) .
$$

Take a positive constant $r_{0}$ such that $r_{0} C<1 / 2$. Thus, if $|\xi-\eta| \leqq r_{0} \lambda_{1}(\xi)$, then $\left|\lambda_{1}(\eta)-\lambda_{1}(\xi)\right| \leqq(1 / 2) \lambda_{1}(\xi)$. Hence we have $1 / 2 \leqq \lambda_{1}(\eta) / \lambda_{1}(\xi) \leqq 3 / 2$. have

COROLlary. Let $\lambda_{\varepsilon}(\xi)$ be a werght function of type $\varepsilon(0 \leqq \varepsilon<1)$. Then we

(i) If $|\xi-\eta| \leqq \gamma_{0} \lambda_{\varepsilon}(\xi)$, then $C^{-1} \leqq \lambda_{\varepsilon}(\eta) / \lambda_{\varepsilon}(\xi) \leqq C$.

(ii) If $|\xi-\sigma| \leqq r_{0} \lambda_{\varepsilon}(\sigma)$ and $\left|\xi-\sigma^{\prime}\right| \leqq r_{0} \lambda_{\varepsilon}\left(\sigma^{\prime}\right)$, then

$$
C^{-1} \leqq \lambda_{\varepsilon}\left(\sigma^{\prime}\right) / \lambda_{\varepsilon}(\sigma) \leqq C \text {. }
$$

(iii) If $\left|\sigma-\sigma^{\prime}\right| \leqq \frac{1}{2} \gamma_{0}\left(\lambda_{\varepsilon}(\sigma)+\lambda_{\varepsilon}\left(\sigma^{\prime}\right)\right)$, then $C^{-1} \leqq \lambda_{\varepsilon}\left(\sigma^{\prime}\right) / \lambda_{\varepsilon}(\sigma) \leqq C$.

From now on we fix a weight function $\lambda_{\varepsilon}(\xi)$ of type $\varepsilon(0 \leqq \varepsilon<1)$ and we omit the subscript $\varepsilon$ and write $\lambda(\xi)$.

Let $r$ be a positive real number. We set

$$
U_{(s, \sigma)}(r)=\left\{(x, \xi) ;|x-s| \leqq r \lambda(\sigma)^{-1},|\xi-\sigma| \leqq r \lambda(\sigma)\right\}
$$

for $(s, \sigma)$ in $\boldsymbol{R}^{n} \times \boldsymbol{R}^{n}$. This set is a neighborhood of $(s, \sigma)$ in $\boldsymbol{R}^{n} \times \boldsymbol{R}^{n}$, where we endow a Riemannian metric at $(s, \sigma)$ as follows 


$$
g_{(s, \sigma)}(x, \xi)=\lambda(\sigma)^{2}|x|^{2}+\lambda(\sigma)^{-2}|\xi|^{2} .
$$

This Riemannian metric $g_{(s, \sigma)}$ is slowly varying, $g \leqq g^{\sigma}$ and $\sigma$-temperate in the sense of Hörmander [9].

Remark 4. Corollary of Lemma 2 implies that $C^{-1} \leqq \lambda(\xi) / \lambda(\sigma) \leqq C$ for all $(x, \xi)$ in $U_{(s, \sigma)}\left(r_{0}\right)$.

We shall construct a partition of unity with contınuous parameters subordinated to a covering $\left\{U_{(s, \sigma)}(r)\right\}_{(s, \sigma) \in \boldsymbol{R}^{n} \times \boldsymbol{R}^{n}}$ (for some $\left.r>0\right)$ of $\boldsymbol{R}^{n} \times \boldsymbol{R}^{n}$ which a weight function $\lambda(\xi)$ defines. This partition of unity is similar to that in Hörmander [9] which depends on discrete parameters.

LEMMA 3. Let $r_{1}$ and $r_{2}$ be real numbers such that $0<r_{2}<r_{1}<(1 / 4) r_{0}$. Then we can choose $C^{\infty}$-functions $\varphi_{(s, \sigma)}(x, \xi)$ contrnuously depending on $(s, \sigma)$ in $\boldsymbol{R}^{n} \times \boldsymbol{R}^{n}$ and satisfying the following conditions:

(i) Each $\varphi_{(s, \sigma)}(x, \xi)$ is non-negative, structly positive for all $(\lambda, \xi)$ in $U_{(s, \sigma)}\left(r_{2}\right)$ and is supported in $U_{(s, \sigma)}\left(r_{1}\right)$.

$$
\iint_{R^{n_{\times} \boldsymbol{R}^{n}}} \varphi(s, \sigma)(x, \xi) d s d \sigma=1 .
$$

(iii) For any two multi-indices $\alpha$ and $\beta$ there exists a constant $C_{\kappa, \beta}$ such that

$$
\sup _{(x, \xi) \in R^{n} \times R^{n}}\left|\partial_{x}^{\alpha} \partial_{\xi}^{\beta} \varphi_{(s, \sigma)}(x, \xi)\right| \leqq C_{\alpha, \beta} \lambda(\sigma)^{|\alpha|-|\beta|},
$$

where the constant $C_{\alpha, \beta}$ is independent of $(s, \sigma)$.

Proof. Take a $C^{\infty}$ function $\varphi$ in $\boldsymbol{R}^{1}$ such that $0 \leqq \varphi(t) \leqq 1, \varphi(t)=1$ if $t \leqq \gamma_{2}$ and $\varphi(t)=0$ if $t \geqq r_{1}$. And set

$$
\begin{gathered}
\psi_{(s, \sigma)}(x, \xi)=\varphi(\lambda(\sigma)|x-s|) \cdot \varphi\left(\lambda(\sigma)^{-1}|\xi-\sigma|\right), \\
\Psi(x, \xi)=\iint_{R^{n \times R^{n}}} \psi_{(s, \sigma)}(x, \xi) d s d \sigma .
\end{gathered}
$$

First the followings are obvious :

$$
\begin{gathered}
0 \leqq \phi_{(s, \sigma)}(x, \xi) \leqq 1 \\
\operatorname{supp}_{(x, \xi)} \phi_{(s, \sigma)} \subset U_{(s, \sigma)}\left(r_{1}\right)
\end{gathered}
$$

and

$$
\psi_{(s, \sigma)}(x, \xi)=1 \text { whenever }(x, \xi) \text { is in } U_{(s, \sigma)}\left(r_{2}\right) \text {. }
$$

Next we can prove (iii) for $\phi_{(s, \sigma)}(x, \xi)$. By induction we see that

$$
\partial_{x}^{\alpha} \partial_{\xi}^{\beta} \psi_{(s, \sigma)}(x, \xi)=\partial_{x}^{\alpha} \varphi(\lambda(\sigma)|x-s|) \cdot \partial_{\xi}^{\beta} \varphi\left(\lambda(\sigma)^{-1}|\xi-\sigma|\right)
$$

is a sum of terms of the form 


$$
\begin{aligned}
& C \varphi^{(j)}(\lambda(\sigma)|x-s|) \cdot \lambda(\sigma)^{\jmath} \prod_{\mu=1}^{\jmath} \partial_{x}{ }^{\alpha} \mu|x-s| \\
& \quad \times \varphi^{(k)}\left(\lambda(\sigma)^{-1}|\xi-\sigma|\right) \cdot \lambda(\sigma)^{-k} \prod_{\nu=1}^{k} \partial_{\xi} \beta_{\nu}|\xi-\sigma|,
\end{aligned}
$$

where

$$
\begin{aligned}
& 1 \leqq \jmath \leqq|\alpha|, \quad\left|\alpha_{1}\right|+\cdots+\left|\alpha_{j}\right|=|\alpha| \\
& 1 \leqq k \leqq|\beta|, \quad\left|\beta_{1}\right|+\cdots+\left|\beta_{k}\right|=|\beta| .
\end{aligned}
$$

Therefore each term (14) is dominated by

$$
\begin{aligned}
& C_{\alpha, \beta}\left|\varphi^{(\jmath)}(\lambda(\sigma)|x-s|)\right| \lambda(\sigma)^{\jmath} \prod_{\mu=1}^{J}|x-s|^{1-\left|\alpha \mu^{\prime}\right|} \\
& \quad \times\left|\varphi^{(k)}\left(\lambda(\sigma)^{-1}|\xi-\sigma|\right)\right| \lambda(\sigma)^{-k} \prod_{\nu=1}^{k}|\xi-\sigma|^{1-\left|\beta_{\nu}\right|} \\
\leqq & C_{\alpha, \beta}\left(\sum_{j=0}^{|\alpha|} \sup _{t}\left|\varphi^{(\jmath)}(t)\right| t^{\jmath-|\alpha|}\right)\left(\sum_{k=0}^{|\beta|} \sup _{t}\left|\varphi^{(k)}(t)\right| t^{k-|\beta|}\right) \lambda(\sigma)^{|\alpha|-|\beta|} .
\end{aligned}
$$

Thus,

$$
\left|\partial_{x}^{\alpha} \partial_{\xi}^{\beta} \psi_{(s, \sigma)}(x, \xi)\right| \leqq C_{\alpha, \beta} \lambda(\sigma)^{|\alpha|-|\beta|} .
$$

Third we show that the inequalities

$$
C \leqq \Psi(x, \xi) \leqq C^{\prime}
$$

hold for some positive constants $C$ and $C^{\prime}$. We note from Remark 4 that the inequalities

$$
C_{r} \leqq \iint_{R^{n} \times R^{n}} \chi_{r}(\lambda(\sigma)(x-s)) \chi_{r}\left(\lambda(\sigma)^{-1}(\xi-\sigma)\right) d s d \sigma \leqq C_{r}^{\prime}
$$

hold for all $r$ such that $0<r<r_{0}$. Here $\chi_{r}$ denotes the characteristics function of the ball of radius $r$. The properties (11), (12) and (13) imply that

$$
\begin{aligned}
\chi_{r_{2}}(\lambda(\sigma)(x-s)) \chi_{r_{2}}\left(\lambda(\sigma)^{-1}(\xi-\sigma)\right) \leqq & (s, \sigma) \\
& \leqq \chi_{r_{1}}(\lambda(\sigma)(x-s)) \chi_{r_{1}}\left(\lambda(\sigma)^{-1}(\xi-\sigma)\right) .
\end{aligned}
$$

Substituting (18) into (10) and considering (17) we have the inequalities (16).

Fourth, we prove that $\Psi(x, \xi)$ is in $S^{0}(\lambda)$. We differentiate (10) under integral sign and use (15) and (17) in view of (12) and Remark 4 . Thus,

$$
\begin{aligned}
\left|\partial_{x}^{\alpha} \partial_{\xi}^{\beta} \Psi(x, \xi)\right| & \leqq \iint_{R^{n} \times \boldsymbol{R}^{n}}\left|\partial_{x}^{\alpha} \partial_{\xi}^{\beta} \psi_{(s, \sigma)}(x, \xi)\right| d s d \sigma \\
& \leqq C_{\alpha, \beta} \lambda(\sigma)^{|\alpha|-|\beta|} \iint_{\boldsymbol{R}^{n} \times \boldsymbol{R}^{n}} \chi_{r_{1}}(\lambda(\sigma)(x-s)) \chi_{r_{1}}\left(\lambda(\sigma)^{-1}(\xi-\sigma)\right) d s d \sigma \\
& \leqq C_{\alpha, \beta} \lambda(\sigma)^{|\alpha|-|\beta|} .
\end{aligned}
$$

Now we set 


$$
\varphi_{(s, \sigma)}(x, \xi)=\phi_{(s, \sigma)}(x, \xi) / \Psi(x, \xi) .
$$

From (11), (12), (13), (15), (16) and (19) it is clear that $\varphi_{(s . \sigma)}(x, \xi)$ satisfies the required properties.

Let $t=(s, \sigma)$ be any point in $\boldsymbol{R}^{n} \times \boldsymbol{R}^{n}$ and set

$$
a_{t}(x, \xi)=\varphi_{t}(x, \xi) a(x, \xi) \text {. }
$$

Each $a_{t}(x, \xi)$ is supported in a set $U_{t}\left(r_{1}\right)$ and for any two multi-indices $\alpha$ and $\beta$ estimates

$$
\left|\partial_{x}^{\alpha} \partial_{\xi}^{\beta} a_{t}(x, \xi)\right| \leqq C_{\alpha, \beta}|a|_{0 ;|\alpha|+|\beta|} \lambda(\sigma)^{|\alpha|-|\beta|} .
$$

hold for some constants $C_{\alpha, \beta}$. And define

$$
A_{t} f(x)=\int_{R^{n}} e^{\imath S(x, \xi)} a_{t}(x, \xi) f(\xi) d \xi .
$$

Then we have

$$
A f(x)=\int_{R^{2 n}} A_{t} f(x) d t .
$$

The adjoint operator $A_{t^{\prime}}^{*}$ of $A_{t^{\prime}}$ for $t^{\prime}=\left(s^{\prime}, \sigma^{\prime}\right)$ is given by

$$
A_{t^{\prime}}^{*} g(\xi)=\int_{R^{n}} e^{-i S(y, \xi)} \overline{a_{t^{\prime}}(y, \xi)} g(y) d y,
$$

where $\overline{a_{t^{\prime}}(y, \xi)}$ is the complex conjugate of $a_{t^{\prime}}(y, \xi)$.

Now we prepare to apply the lemma of Cotlar-Knapp-Stein formulated by Calderón-Vaillancourt [5] (See Lemma 7 below). Thus we have only to prove all of the following estimates:

$1^{\circ}$ There exists a positive constant $C$ independent of $t=(s, \sigma)$ such that

$$
\left\|A_{t}\right\| \leqq C .
$$

$2^{\circ} \quad$ There exist non-negative functions $h\left(t, t^{\prime}\right)$ and $k\left(t, t^{\prime}\right)$ such that

$$
\left\|A_{t} A_{t^{\prime}}^{*}\right\| \leqq h\left(t, t^{\prime}\right)^{2}, \quad\left\|A_{t}^{*} A_{t^{\prime}}\right\| \leqq k\left(t, t^{\prime}\right)^{2} .
$$

$3^{\circ}$ The above functions satisfy the following estimates

$$
\sup _{t^{\prime}} \int_{R^{2 n}} h\left(t, t^{\prime}\right) d t \leqq M, \quad \sup _{t^{\prime}} \int_{R^{2 n}} k\left(t, t^{\prime}\right) d t \leqq M
$$

for some constant $M$.

Proof of $1^{\circ}$ We know from (20) and (22) that the integral kernel function $H_{t}(x, \xi)$ of $A_{t}$ is dominated by

Thus we have

$$
C|a|_{0,0} \chi_{r_{1}}(\lambda(\sigma)(x-s)) \chi_{r_{1}}\left(\lambda(\sigma)^{-1}(\xi-\sigma)\right) .
$$




$$
\begin{aligned}
& \int_{R^{n}}\left|H_{t}(x, \xi)\right| d x \leqq C_{n, r_{1}}|a|_{0,0} \lambda(\sigma)^{-n}, \\
& \int_{R^{n}}\left|H_{t}(x, \xi)\right| d \xi \leqq C_{n, r_{1}}|a|_{0,0} \lambda(\sigma)^{n} .
\end{aligned}
$$

Hence these estimates imply the desired inequality

$$
\left\|A_{t}\right\| \leqq C_{n, r_{1}}|a|_{0,0} \text {. }
$$

For $t=(s, \sigma)$ and $t^{\prime}=\left(s^{\prime}, \sigma^{\prime}\right)$ we denote the integral kernel functions of the operators $A_{t} A_{t^{\prime}}^{*}$ and $A_{t}^{*} A_{t^{\prime}}$ by $H_{t, t^{\prime}}(x, y)$ and $K_{t, t^{\prime}}(\xi, \eta)$ respectively. Thus, from (22) and (24) we have the following expressions.

$$
A_{t} A_{t^{\prime}}^{*} f(x)=\int_{R^{n}} H_{t, t^{\prime}}(x, y) f(y) d y,
$$

where

$$
H_{t, t^{\prime}}(x, y)=\int_{R^{n}} e^{\imath(S(x, \xi)-S(y, \xi))} a_{t}(x, \xi) \overline{a_{t^{\prime}}(y, \xi)} d \xi
$$

And

$$
A_{t}^{*} A_{t^{\prime}} g(\xi)=\int_{R^{n}} K_{t, t^{\prime}}(\xi, \eta) g(\eta) d \eta,
$$

where

$$
K_{t t^{\prime}}(\xi, \eta)=\int_{R^{n}} e^{-\imath(S(x, \xi)-S(x, \eta))} \overline{a_{t}(x, \xi)} a_{t^{\prime}}(x, \eta) d x .
$$

Now we shall estimate $H_{t, t^{\prime}}(x, y)$ and $K_{t, t^{\prime}}(\xi, \eta)$ in the following Proposition 1. Then we need two lemmas concerning the phase functions and integration by parts (See Lemmas 4 and 5 below). And we shall prove the statements $2^{\circ}$ and $3^{\circ}$ as Propositions 2 and 3 respectively.

LEMMA 4. 1) There exists a positive constant $\delta_{1}$ such that

$$
\left|\nabla_{\xi}(S(x, \xi)-S(y, \xi))\right| \geqq \delta_{1}|x-y|
$$

and

$$
\left|\nabla_{x}(S(x, \xi)-S(x, \eta))\right| \geqq \delta_{1}|\xi-\eta| .
$$

2-i) For any multi-index $\alpha$ such that $|\alpha| \geqq 1$ there exists a constant $C_{\alpha}$ such that the estrmates

$$
\left(\lambda(\sigma)^{-1}+\lambda\left(\sigma^{\prime}\right)^{-1}\right)^{-|\alpha|}\left|\partial_{\xi}^{\alpha}(S(x, \xi)-S(y, \xi))\right| \leqq C_{\alpha} \rho
$$

hold for all $(x, \xi)$ in $U_{(s, \sigma)}\left(r_{1}\right)$ and $(y, \xi)$ in $U_{\left(s^{\prime}, \sigma^{\prime}\right)}\left(r_{1}\right)$, where

$$
\rho=\left\{1+\left(\lambda(\sigma)^{-1}+\lambda\left(\sigma^{\prime}\right)^{-1}\right)^{-2}\left|\nabla_{\xi}(S(x, \xi)-S(y, \xi))\right|^{2}\right\}^{1 / 2} .
$$


2-ii) For any multı-index $\alpha$ such that $|\alpha| \geqq 1$ there exists a constant $C_{\alpha}$ such that the estimates

$$
\left(\lambda(\sigma)+\lambda\left(\sigma^{\prime}\right)\right)^{-|\alpha|}\left|\partial_{x}^{\alpha}(S(x, \xi)-S(x, \eta))\right| \leqq C_{\alpha} \tau
$$

hold for all $(x, \xi)$ in $U_{(s, \sigma)}\left(r_{1}\right)$ and $(x, \eta)$ in $U_{\left(s^{\prime}, \sigma^{\prime}\right)}\left(r_{1}\right)$, where

$$
\tau=\left\{1+\left(\lambda(\sigma)+\lambda\left(\sigma^{\prime}\right)\right)^{-2} \mid \nabla_{x}\left(S(x, \xi)-\left.S(x, \eta)\right|^{2}\right\}^{1 / 2} .\right.
$$

Proof. 1) Let $z=\nabla_{\xi} S(x, \xi)$ and $w=\nabla_{\xi} S(y, \xi)$. Because of (P-1) and (P-2), we can apply the global implicit function theorem to the mapping

$$
T_{\xi}: \boldsymbol{R}^{n} \ni x \longrightarrow z=\nabla_{\xi} S(x, \xi) \in \boldsymbol{R}^{n},
$$

where $\xi \in \boldsymbol{R}^{n}$ is fixed. Thus, $T_{\xi}$ is a global diffeomorphism. When we consider $x$ as a function of $(z, \xi)$, we write $x=x(z, \xi)$. Since the Jacobian matrix $\partial x / \partial z$ is the inverse matrix of $\left(\partial_{x_{j}} \partial_{\xi_{k}} S(x, \xi)\right)$, each component of $\partial x / \partial z$ has an upper bound $\gamma=C_{n} C_{1,1}^{n-1} \delta_{0}^{-1}$. By the mean value theorem we obtain

Thus,

$$
|x(z, \xi)-x(w, \xi)| \leqq \gamma|z-w| .
$$

$$
|x-y| \leqq \gamma\left|\nabla_{\xi} S(x, \xi)-\nabla_{\xi} S(y, \xi)\right| \text {. }
$$

This is equivalent to the inequality (29) with $\delta_{1}=\gamma^{-1}=\delta_{0} / C_{n} C_{1,1}^{n-1}$. A similar argument shows that the inequality (30) is valid.

2) When $|\alpha|=1$, the inequality (31) is valid from the definition of $\rho$. When $|\alpha| \geqq 2$, we have, for $(x, \xi)$ in $U_{(s, \sigma)}\left(r_{1}\right)$ and $(y, \xi)$ in $U\left({ }_{\left(s^{\prime}, \sigma^{\prime}\right)}\left(r_{1}\right)\right.$,

Then

$$
\left|\partial_{\xi}^{\alpha} S(x, \xi)\right| \leqq C_{\alpha} \lambda(\sigma)^{-|\alpha|}, \quad\left|\partial_{\xi}^{\alpha} S(y, \xi)\right| \leqq C_{\alpha} \lambda\left(\sigma^{\prime}\right)^{-|\alpha|} .
$$

$$
\begin{aligned}
\left(\lambda(\sigma)^{-1}+\right. & \left.\lambda\left(\sigma^{\prime}\right)^{-1}\right)^{-|\alpha|}\left|\partial_{\xi}^{\alpha} S(x, \xi)-\partial_{\xi}^{\alpha} S(y, \xi)\right| \\
& \leqq C_{\alpha}\left(\lambda(\sigma)^{-1}+\lambda\left(\sigma^{\prime}\right)^{-1}\right)^{-|\alpha|}\left(\lambda(\sigma)^{-|\alpha|}+\lambda\left(\sigma^{\prime}\right)^{-|\alpha|}\right) \\
& \leqq 2 C_{\alpha} \leqq 2 C_{\alpha} \rho .
\end{aligned}
$$

Thus, the inequality (31) is valid. By a similar argument we know that the second inequality (33) is also valid. This completes the proof of Lemma 4.

Lemma 5. Let $L$ be a partial differential operator of order 1:

$$
L u(x)=\rho^{-2}\left(1-\imath K^{-2} \nabla_{x} F(x) \cdot \nabla_{x}\right) u(x),
$$

where $K$ is a positive constant, $F(x)$ is a smooth real-valued function and

Then,

$$
\rho=\left(1+K^{-2}\left|\nabla_{x} F(x)\right|^{2}\right)^{1 / 2} .
$$

$$
L e^{\imath F(x)}=e^{i F(x)} .
$$


(ii) We denote by ${ }^{t} L$ the formal transposed operator of $L$. Then for any positive integer $m,\left({ }^{t} L\right)^{m} u(x)$ is a sum of terms of the form

$$
C \rho^{-k}\left\{\prod_{\nu=1}^{q} K^{-1 \alpha_{\nu}} \partial_{x}^{\alpha_{\nu}} F(x)\right\} K^{-|\beta|} \partial_{x}^{\beta} u(x),
$$

where

$$
\begin{aligned}
& 2 m \leqq k \leqq 4 m, \quad k-2 m \leqq q \leqq k-m, \\
& \left|\alpha_{\nu}\right| \geqq 1, \quad \sum_{\nu=1}^{q}\left|\alpha_{\nu}\right| \leqq q+m, \quad|\beta| \leqq m .
\end{aligned}
$$

Proof. We use the same procedure as the proof of Lemma 2.5 in AsadaFujiwara [1, p. 331].

The identity (i) follows from definition of $L$ and $\rho$.

To prove (ii) we note that

$$
\partial_{x_{j}} \rho^{-m}=-m \rho^{-m-2} K^{-2} \sum_{k=1}^{n} \partial_{x_{k}} F \cdot \partial_{x_{j}} \partial_{x_{k}} F .
$$

Then Leibniz's rule shows that

$$
\begin{aligned}
{ }^{t} L u(x)= & \rho^{-2} u(x)+\imath K^{-2} \sum_{j=1}^{n} \partial_{x_{j}}\left(\rho^{-2} \partial_{x_{j}} F(x) \cdot u(x)\right) \\
= & \rho^{-2} u(x)+i(-2) \rho^{-4} K^{-4} \sum_{j, k=1}^{n} \partial_{x_{j}} F \cdot \partial_{x_{k}} F \cdot \partial_{x_{j}} \partial_{x_{k}} F \cdot u(x) \\
& +\imath \rho^{-2} K^{-2} \sum_{j=1}^{n} \partial_{x_{j}}^{2} F \cdot u(x)+i \rho^{-2} K^{-2} \sum_{j=1}^{n} \partial_{x_{j}} F \cdot \partial_{x_{j}} u .
\end{aligned}
$$

Thus ${ }^{t} \mathrm{~L}$ is a linear combination of operators of the form

$$
\begin{aligned}
& \rho^{-2} \times \\
& \rho^{-4} K^{-4} \partial_{x_{j}} F \cdot \partial_{x_{k}} F \cdot \partial_{x_{j}} \partial_{x_{k}} F \times, \\
& \rho^{-2} K^{-2} \partial_{x_{j}}^{2} F \times, \\
& \rho^{-2} K^{-2} \partial_{x_{j}} F \cdot \partial_{x_{j}} .
\end{aligned}
$$

Now we say that the term (35) is of the type $\left(k, q, \sum_{\nu=1}^{q}\left|\alpha_{\nu}\right|,|\beta|\right)$. Then ${ }^{t} L u$ is a sum of terms of the types $(2,0,0,0),(4,3,4,0),(2,1,2,0)$ and $(2,1,1,1)$. When we operate (37), (38) and (39) to a term (35) of the type $\left(k, q, \Sigma\left|\alpha_{\nu}\right|,|\beta|\right)$ once, the type of the resultant term increases by $(2,0,0,0)$, $(4,3,4,0)$ and $(2,1,2,0)$, respectively. Next we examine how the types change when we operate an operator (40) to a term (35). Leibniz's rule shows that

$$
\begin{aligned}
& \rho^{-2} K^{-2} \partial_{x_{j}} F \cdot \partial_{x_{j}}\left(\rho^{-k} \cdot \prod_{\nu=1}^{q} K^{-\left|\alpha_{\nu}\right|} \partial_{x}^{\alpha_{\nu}} F(x) \cdot K^{-|\beta|} \partial_{x}^{\beta} u(x)\right) \\
& =(-k) \rho^{-(k+4)} K^{-4} \sum_{\imath=1}^{n} \partial_{x_{\imath}} F \cdot \partial_{x_{j}} F \cdot \partial_{x_{\imath}} \partial_{x_{j}} F \prod_{\nu=1}^{q} K^{-\left|\alpha_{\nu}\right|} \partial_{x}^{\alpha_{\nu}} F \times K^{-|\beta|} \partial_{x}^{\beta} u(x)
\end{aligned}
$$




$$
\begin{aligned}
& +\rho^{-(k+2)} K^{-1} \partial_{x_{j}} F \times \sum_{\mu=1}^{q}\left(\prod_{\nu \neq \mu} K^{-\mid \alpha_{\nu}} \partial_{x}^{\alpha_{\nu}} F\right) K^{-1} \partial_{x_{j}} K^{-\mid \alpha \mu^{\prime}} \partial_{x}^{\alpha} \mu F \times K^{-|\beta|} \partial_{x}^{\beta} u(x) \\
& +\rho^{-(k+2)} K^{-1} \partial_{x_{j}} F \times \prod_{\nu=1}^{q} K^{-\left|\alpha_{\nu}\right|} \partial_{x}^{\alpha_{\nu}} F(x) \times K^{-1} \partial_{x_{j}}\left(K^{-|\beta|} \partial_{x}^{\beta} u(x)\right) .
\end{aligned}
$$

The resultant terms under operations of (40) are a sum of terms the types of which increase by $(4,3,4,0),(2,1,2,0)$ and $(2,1,1,1)$. Consequently, when we operate ${ }^{t} L$ to a term (35), the types of the resultant terms increase by $(2,0,0,0)$, $(4,3,4,0),(2,1,2,0)$ and $(2,1,1,1)$. We repeat the process; thus we have

$$
\left({ }^{t} L\right)^{m} u(x)=\Sigma C \rho^{-k} \prod_{\nu=1}^{q} K^{-\left|\alpha_{\nu}\right|} \partial_{x}^{\alpha_{\nu}} F(x) \times K^{-1 \beta} \mid \partial_{x}^{\beta} u(x) .
$$

Here the summation is taken all over non-negative integers $i_{1}, \imath_{2}, \imath_{3}, \imath_{4}$ such that $i_{1}+i_{2}+i_{3}+i_{4}=m$. And

$$
\left(k, q, \Sigma\left|\alpha_{\nu}\right|,|\beta|\right)=\imath_{1}(2,0,0,0)+\imath_{2}(4,3,4,0)+i_{3}(2,1,2,0)+\imath_{4}(2,1,1,1) .
$$

Then $k, q, \alpha_{\nu}, \beta$ satisfy the condition (36). This completes the proof of Lemma 5 .

Now using Lemmas 4 and 5 we obtain estimates for the integral kernel functions $H_{t, t^{\prime}}(x, y)$ and $K_{t, t^{\prime}}(\xi, \eta)$, where $t=(s, \sigma)$ and $t^{\prime}=\left(s^{\prime}, \sigma^{\prime}\right)$ are parameters in $\boldsymbol{R}^{n} \times \boldsymbol{R}^{n}$.

Proposition 1. 1) For any non-negative integer $m$ there exists a constant $C_{m}$ such that

$$
\begin{aligned}
\left|H_{t, t^{\prime}}(x, y)\right| \leqq C_{m}|a|_{0 ; m}^{2} \min \left\{\lambda(\sigma), \lambda\left(\sigma^{\prime}\right)\right\}{ }^{n} \chi_{r_{1}}\left(\frac{\sigma-\sigma^{\prime}}{\lambda(\sigma)+\lambda\left(\sigma^{\prime}\right)}\right) \\
\times \frac{\chi_{r_{1}}(\lambda(\sigma)(x-s)) \chi_{r_{1}}\left(\lambda\left(\sigma^{\prime}\right)\left(y-s^{\prime}\right)\right)}{\left(1+\delta_{1}^{2}\left(\lambda(\sigma)^{-1}+\lambda\left(\sigma^{\prime}\right)^{-1}\right)^{-2}|x-y|^{2}\right)^{m / 2}},
\end{aligned}
$$

where $\chi_{r}$ is the characterisrac function of the ball $\{x ;|x| \leqq r\}$. And the above constant $C_{m}$ is independent of $x, y, t=(s, \sigma)$ and $t^{\prime}=\left(s^{\prime}, \sigma^{\prime}\right)$.

2) For any non-negative integer $m$ there exists a constant $C_{m}$ such that

$$
\begin{aligned}
\left|K_{t, t^{\prime}}(\xi, \eta)\right| \leqq C_{m}|a|_{0, m}^{2} \min \left\{\lambda(\sigma)^{-1}, \lambda\left(\sigma^{\prime}\right)^{-1}\right\}^{n} \chi_{r_{1}}\left(\frac{s-s^{\prime}}{\lambda(\sigma)^{-1}+\lambda\left(\sigma^{\prime}\right)^{-1}}\right) \\
\times \frac{\chi_{r_{1}}\left(\lambda(\sigma)^{-1}(\xi-\sigma)\right) \chi_{r_{1}}\left(\lambda\left(\sigma^{\prime}\right)^{-1}\left(\eta-\sigma^{\prime}\right)\right)}{\left(1+\delta_{1}^{2}\left(\lambda(\sigma)+\lambda\left(\sigma^{\prime}\right)\right)^{-2}|\xi-\eta|^{2}\right)^{m / 2}},
\end{aligned}
$$

where $C_{m}$ is independent of $x, y, t=(s, \sigma)$ and $t^{\prime}=\left(s^{\prime}, \sigma^{\prime}\right)$.

Proof. 1) We set

$$
F(\xi, x, y)=S(x, \xi)-S(y, \xi) .
$$

Then from (26) we have

$$
H_{t, t^{\prime}}(x, y)=\int_{R^{n}} e^{\imath F(\xi, x, y)} a_{t}(x, \xi) \overline{a_{t^{\prime}}(y, \xi)} d \xi .
$$


Let $L$ be a partial differential operator of order 1 :

where

$$
L=\rho^{-2}\left\{1-i\left(\lambda(\sigma)^{-1}+\lambda\left(\sigma^{\prime}\right)^{-1}\right)^{-2} \nabla_{\xi} F(\xi, x, y) \cdot \nabla_{\xi}\right\},
$$

$$
\rho=\left\{1+\left(\lambda(\sigma)^{-1}+\lambda\left(\sigma^{\prime}\right)^{-1}\right)^{-2}\left|\nabla_{\xi} F\right|^{2}\right\}^{1 / 2} .
$$

Then we rewrite the right-hand side of (43) using the identity ( $i$ ) of Lemma 5 and integrate by parts, and repeat the process; thus we have

$$
H_{t, t^{\prime}}(x, y)=\int_{R^{n}} e^{i F(\xi, x, y)\left({ }^{t} L\right)^{m}}\left[a_{t}(x, \xi) \overline{a_{t^{\prime}}(y, \xi)}\right] d \xi,
$$

where $m$ is an arbitrary non-negative integer. Applying (ii) of Lemma 5 we see that $\left({ }^{t} L\right)^{m}\left(a_{t} \overline{a_{t^{\prime}}}\right)$ is a sum of terms of the form

$$
C \rho^{-k}\left(\lambda(\sigma)^{-1}+\lambda\left(\sigma^{\prime}\right)^{-1}\right)^{-\jmath} \cdot \prod_{\nu=1}^{q} \partial_{\xi}^{\alpha} F \cdot \partial_{\xi}^{\beta}\left(a_{t} \overline{a_{t^{\prime}}}\right),
$$

where

$$
\begin{aligned}
& 2 m \leqq k \leqq 4 m, \quad k-2 m \leqq q \leqq k-m, \\
& \left|\alpha_{\nu}\right| \geqq 1, \quad q \leqq \sum_{\nu=1}^{q}\left|\alpha_{\nu}\right| \leqq q+m, \quad|\beta| \leqq m, \\
& \jmath=\sum_{\nu=1}^{q}\left|\alpha_{\nu}\right|+|\beta| .
\end{aligned}
$$

Leibniz's rule and estimates (21) show that

$$
\left|\partial_{\xi}^{\beta}\left(a_{t} \overline{a_{t^{\prime}}}\right)\right| \leqq C_{\beta}|a|_{0,|\beta|}^{2}\left(\lambda(\sigma)^{-1}+\lambda\left(\sigma^{\prime}\right)^{-1}\right)^{|\beta|} .
$$

Estimates (31) of Lemma 4, (46) and (47) show that each term (45) is dominated by

$$
\begin{aligned}
& C_{m} \rho^{-k} \sum_{\nu=1}^{q} \frac{\left|\partial_{\xi}^{\alpha_{\nu}} F\right|}{\left(\lambda(\sigma)^{-1}+\lambda\left(\sigma^{\prime}\right)^{-1}\right)^{\left|\alpha_{\nu}\right|}} \cdot \frac{\left|\partial_{\xi}^{\beta}\left(a_{t} \overline{a_{t^{\prime}}}\right)\right|}{\left(\lambda(\sigma)^{-1}+\lambda\left(\sigma^{\prime}\right)^{-1}\right)^{|\beta|}} \\
& \leqq C_{m} \rho^{-k+q}|a|_{0, m}^{2} \leqq C_{m} \rho^{-m}|a|_{0}^{2}, m
\end{aligned}
$$

Thus,

$$
\left.\mid{ }^{t} L\right)\left.^{m}\left(a_{t} \overline{a_{t^{\prime}}}\right)\left|\leqq C_{m} \rho^{-m}\right| a\right|_{0, m} ^{2} .
$$

When we apply estimates (48) and (29) of Lemma 4 to the right-hand side of (44), considering the support of the integrand in it, we have

$$
\begin{aligned}
\left|H_{t, t^{\prime}}(x, y)\right| \leqq C_{m} \mid & \left.a\right|_{0, m} ^{2} \frac{\chi_{r_{1}}(\lambda(\sigma)(x-s)) \chi_{r_{1}}\left(\lambda\left(\sigma^{\prime}\right)(y-s)\right)}{\left\{1+\delta_{1}^{2}\left(\lambda(\sigma)^{-1}+\lambda\left(\sigma^{\prime}\right)^{-1}\right)^{-2}|x-y|^{2}\right\}^{m / 2}} \\
& \times \int_{R^{n}} \chi_{r_{1}}\left(\lambda(\sigma)^{-1}(\xi-\sigma)\right) \chi_{r_{1}}\left(\lambda\left(\sigma^{\prime}\right)^{-1}\left(\xi-\sigma^{\prime}\right)\right) d \xi .
\end{aligned}
$$

We note that 


$$
\begin{aligned}
& \int_{R^{n}} \chi_{r_{1}}\left(\lambda(\sigma)^{-1}(\xi-\sigma)\right) \chi_{r_{1}}\left(\lambda\left(\sigma^{\prime}\right)^{-1}\left(\xi-\sigma^{\prime}\right)\right) d \xi \\
& \leqq C_{n, r_{1}} \min \left\{\lambda(\sigma), \lambda\left(\sigma^{\prime}\right)\right\}^{n} \chi_{r_{1}}\left(\frac{\sigma-\sigma^{\prime}}{\lambda(\sigma)+\lambda\left(\sigma^{\prime}\right)}\right) .
\end{aligned}
$$

Therefore we have

$$
\begin{aligned}
\left|H_{t, t^{\prime}}(x, y)\right| \leqq C_{m, n, r_{1}} \mid & \left.a\right|_{0, m} ^{2} \min \left\{\lambda(\sigma), \lambda\left(\sigma^{\prime}\right)\right\}^{n} \chi_{r_{1}}\left(\frac{\sigma-\sigma^{\prime}}{\lambda(\sigma)+\lambda\left(\sigma^{\prime}\right)}\right) \\
& \times \frac{\chi_{r_{1}}(\lambda(\sigma)(x-s)) \chi_{r_{1}}\left(\lambda\left(\sigma^{\prime}\right)\left(y-s^{\prime}\right)\right)}{\left\{1+\delta_{1}^{2}\left(\lambda(\sigma)^{-1}+\lambda\left(\sigma^{\prime}\right)^{-1}\right)^{-2}|x-y|^{2}\right\}^{m / 2}} .
\end{aligned}
$$

This proves the estimate (41).

$2)$ Set $G(x, \xi, \eta)=S(x, \xi)-S(x, \eta)$ and

where

$$
L=\tau^{-2}\left\{1-i\left(\lambda(\sigma)+\lambda\left(\sigma^{\prime}\right)\right)^{-2} \nabla_{x} G \cdot \nabla_{x}\right\},
$$

$$
\tau=\left\{1+\left(\lambda(\sigma)+\lambda\left(\sigma^{\prime}\right)\right)^{-2}\left|\nabla_{x} G\right|^{2}\right\}^{1 / 2} .
$$

Then integrating by parts in (28), we obtain

$$
\left.K_{t, t^{\prime}}(\xi, \eta)=\int_{R^{n}} e^{i G(x, \xi, \eta)(t} L\right)^{m}\left(\overline{a_{t}(x, \xi)} a_{t^{\prime}}(x, \eta)\right) d x .
$$

By Lemma 5-2) and Leibniz's rule we have the estimate

$$
\left.\mid\left({ }^{t} L\right)^{m}\left(\overline{a_{t}(x, \bar{\xi}}\right) a_{t^{\prime}}(x, \eta)\right)\left.\left|\leqq C_{m}\right| a\right|_{0, m} ^{2} \tau^{-m} .
$$

Thus, noting the support of the integrand and using the estimate (30) in Lemma 4 we have

$$
\begin{array}{r}
\left.\left|K_{t, t^{\prime}}(\xi, \eta)\right| \leqq \int_{R^{n}} \mid{ }^{t} L\right)^{m}\left(\overline{a_{t}(x, \xi)} a_{t^{\prime}}(x, \eta)\right) \mid d x \\
\leqq C_{m}|a|_{0}^{2}, \frac{\chi_{r_{1}}\left(\lambda(\sigma)^{-1}(\xi-\sigma)\right) \chi_{r_{1}}\left(\lambda\left(\sigma^{\prime}\right)^{-1}\left(\eta-\sigma^{\prime}\right)\right)}{\left\{1+\delta_{1}^{2}\left(\lambda(\sigma)+\lambda\left(\sigma^{\prime}\right)\right)^{-2}|\xi-\eta|^{2}\right\}^{m / 2}} \\
\quad \times \int_{R^{n}} \chi_{r_{1}}(\lambda(\sigma)(x-s)) \chi_{r_{1}}\left(\lambda\left(\sigma^{\prime}\right)\left(x-s^{\prime}\right)\right) d x \\
\leqq C_{m}|a|_{0, m}^{2} \min \left\{\lambda(\sigma)^{-1}, \lambda\left(\sigma^{\prime}\right)^{-1}\right\}^{n} \chi_{r_{1}}\left(\frac{s-s^{\prime}}{\lambda(\sigma)^{-1}+\lambda\left(\sigma^{\prime}\right)^{-1}}\right) \\
\quad \times \frac{\chi_{r_{1}}\left(\lambda(\sigma)^{-1}(\xi-\sigma)\right) \chi_{r_{1}}\left(\lambda\left(\sigma^{\prime}\right)^{-1}\left(\eta-\sigma^{\prime}\right)\right)}{\left\{1+\delta_{1}^{2}\left(\lambda(\sigma)+\lambda\left(\sigma^{\prime}\right)\right)^{-2}|\xi-\eta|^{2}\right\}^{m / 2}} .
\end{array}
$$

This completes the proof of Proposition 1.

Next we obtain the estimates of the $L^{1}$-norms of $H_{t, t^{\prime}}(x, y)$ and $K_{t, t^{\prime}}(\xi, \eta)$ with respect to the first and second variables, respectively.

Proposition 2. 1) Let $m$ be a non-negative integer. Set 


$$
\begin{array}{r}
h_{1}\left(t, t^{\prime}\right)=C_{m}|a|_{0 ; m} \chi_{r_{1}}\left(\frac{\sigma-\sigma^{\prime}}{\lambda(\sigma)+\lambda\left(\sigma^{\prime}\right)}\right) \chi_{r_{1}}\left(\frac{s-s^{\prime}}{2\left(\lambda(\sigma)^{-1}+\lambda\left(\sigma^{\prime}\right)^{-1}\right.}\right), \\
h_{2}\left(t, t^{\prime}\right)=C_{m}|a|_{0, m} \chi_{r_{1}}\left(\frac{\sigma-\sigma^{\prime}}{\lambda(\sigma)+\lambda\left(\sigma^{\prime}\right)}\right) \\
\quad \times \frac{1-\chi_{r_{1}}\left(\frac{s-s^{\prime}}{2\left(\lambda(\sigma)^{-1}+\lambda\left(\sigma^{\prime}\right)^{-1}\right)}\right)}{\left\{1+\frac{1}{4} \delta_{1}^{2}\left(\lambda(\sigma)^{-1}+\lambda\left(\sigma^{\prime}\right)^{-1}\right)^{-2}\left|s-s^{\prime}\right|^{2}\right\}^{m / 4}}
\end{array}
$$

and

$$
h\left(t, t^{\prime}\right)=h_{1}\left(t, t^{\prime}\right)+h_{2}\left(t, t^{\prime}\right) .
$$

Then we have the estimates

$$
\sup _{y} \int_{R^{n}}\left|H_{t, t^{\prime}}(x, y)\right| d x \leqq h\left(t, t^{\prime}\right)^{2}
$$

and

$$
\sup _{x} \int_{R^{n}}\left|H_{t, t^{\prime}}(x, y)\right| d y=h\left(t, t^{\prime}\right)^{2},
$$

where the constant $C_{m}$ is independent of $t=(s, \sigma)$ and $t^{\prime}=\left(s^{\prime}, \sigma^{\prime}\right)$.

2) For any positive integer $m$ we set

$$
\begin{aligned}
k_{1}\left(t, t^{\prime}\right)=C_{m}|a|_{0, m} \chi_{r_{1}}\left(\frac{s-s^{\prime}}{\lambda(\sigma)^{-1}+\lambda\left(\sigma^{\prime}\right)^{-1}}\right) \chi_{r_{1}}\left(\frac{\sigma-\sigma^{\prime}}{2\left(\lambda(\sigma)+\lambda\left(\sigma^{\prime}\right)\right)}\right), \\
k_{2}\left(t, t^{\prime}\right)=C_{m}|a|_{0, m} \chi_{r_{1}}\left(\frac{s-s^{\prime}}{\lambda(\sigma)^{-1}+\lambda\left(\sigma^{\prime}\right)^{-1}}\right) \\
\times \frac{1-\chi_{r_{1}}\left(\frac{\sigma-\sigma^{\prime}}{2\left(\lambda(\sigma)+\lambda\left(\sigma^{\prime}\right)\right)}\right)}{\left\{1+\frac{1}{4} \delta_{1}^{2}\left(\lambda(\sigma)+\lambda\left(\sigma^{\prime}\right)\right)^{-2}\left|\sigma-\sigma^{\prime}\right|^{2}\right\}^{m / 4}}
\end{aligned}
$$

and

$$
k\left(t, t^{\prime}\right)=k_{1}\left(t, t^{\prime}\right)+k_{2}\left(t, t^{\prime}\right) .
$$

Then we have the estimates

$$
\sup _{\eta} \int_{R^{n}}\left|K_{t, t^{\prime}}(\xi, \eta)\right| d \xi \leqq k\left(t, t^{\prime}\right)^{2}
$$

and

$$
\sup _{\xi} \int_{R^{n}}\left|K_{t, t^{\prime}}(\xi, \eta)\right| d \eta \leqq k\left(t, t^{\prime}\right)^{2} .
$$

Here $C_{m}$ is some constant independent of $t=(s, \sigma)$ and $t^{\prime}=\left(s^{\prime}, \sigma^{\prime}\right)$.

Corollary of Proposition 2. W'e have the followng estimates 


$$
\begin{gathered}
\left\|A_{t} A_{t^{\prime}}^{*}\right\|^{1 / 2} \leqq h\left(t, t^{\prime}\right), \\
\left\|A_{t}^{*} A_{t^{\prime}}\right\|^{1 / 2} \leqq k\left(t, t^{\prime}\right) .
\end{gathered}
$$

Proof of Corollary. We apply Schur's lemma. Thus the estimate (58) follows from (52) and (53). And the estimate (59) follows from (56) and (57).

Proof of Proposition 2. 1) We consider separately two cases:

$$
\begin{aligned}
& \left|s-s^{\prime}\right| \leqq 2 r_{1}\left(\lambda(\sigma)^{-1}+\lambda\left(\sigma^{\prime}\right)^{-1}\right), \\
& \left|s-s^{\prime}\right| \geqq 2 r_{1}\left(\lambda(\sigma)^{-1}+\lambda\left(\sigma^{\prime}\right)^{-1}\right) .
\end{aligned}
$$

First we work out the case (60). Take $m=0$ in (41). Then

$$
\begin{aligned}
\left|H_{t, t^{\prime}}(x, y)\right| \leqq C_{0}|a|_{0,0}^{2} \min \left\{\lambda(\sigma), \lambda\left(\sigma^{\prime}\right)\right\}^{n} \\
\quad \times \chi_{r_{1}}\left(\frac{\sigma-\sigma^{\prime}}{\lambda(\sigma)+\lambda\left(\sigma^{\prime}\right)}\right) \chi_{r_{1}}(\lambda(\sigma)(x-s)) \chi_{r_{1}}\left(\lambda\left(\sigma^{\prime}\right)\left(y-s^{\prime}\right)\right) .
\end{aligned}
$$

Integration of $(60)$ in $x$ yields

$$
\int_{R^{n}}\left|H_{t, t^{\prime}}(x, y)\right| d x \leqq C_{0, n, r_{1}}|a|_{0,0}^{2} \chi_{r_{1}}\left(\frac{\sigma-\sigma^{\prime}}{\lambda(\sigma)+\lambda\left(\sigma^{\prime}\right)}\right) .
$$

We pass to the non-trivial case (61). We know from (41) that

$$
\lambda(\sigma)|x-s| \leqq r_{1} \text { and } \lambda\left(\sigma^{\prime}\right)\left|y-s^{\prime}\right| \leqq r_{1}
$$

whenever $(x, y)$ is in the support of $H_{t, t^{\prime}}$. Therefore in the case of (61) we have

$$
\begin{aligned}
\left|s-s^{\prime}\right| & \leqq|s-x|+|x-y|+\left|y-s^{\prime}\right| \\
& \leqq\left(\lambda(\sigma)^{-1}+\lambda\left(\sigma^{\prime}\right)^{-1}\right) r_{1}+|x-y| \\
& \leqq \frac{1}{2}\left|s-s^{\prime}\right|+|x-y| .
\end{aligned}
$$

Thus,

$$
\frac{1}{2}\left|s-s^{\prime}\right| \leqq|x-y| \text {. }
$$

Substitution of this inequality (64) into the right-hand side of (41) yields

$$
\begin{aligned}
\left|H_{t, t^{\prime}}(x, y)\right| \leqq C_{m}|a|_{0, m}^{2} \min \left\{\lambda(\sigma), \lambda\left(\sigma^{\prime}\right)\right\}^{n} \\
\times \frac{\chi_{r_{1}}\left(\frac{\sigma-\sigma^{\prime}}{\lambda(\sigma)+\lambda\left(\sigma^{\prime}\right)}\right) \chi_{r_{1}}(\lambda(\sigma)(x-s)) \chi_{r_{1}}\left(\lambda\left(\sigma^{\prime}\right)\left(y-s^{\prime}\right)\right)}{\left\{1+\frac{1}{4} \delta_{1}^{2}\left(\lambda(\sigma)^{-1}+\lambda\left(\sigma^{\prime}\right)^{-1}\right)^{-2}\left|s-s^{\prime}\right|^{2}\right\}^{m / 2}}
\end{aligned}
$$

Then integration of (65) in $x$ shows that 


$$
\begin{aligned}
\int_{R^{n}} \mid & \left.H_{t, t^{\prime}}(x, y)\left|d x \leqq C_{m}\right| a\right|_{0, m} ^{2} \chi_{r_{1}}\left(\frac{\sigma-\sigma^{\prime}}{\lambda(\sigma)+\lambda\left(\sigma^{\prime}\right)}\right) \\
& \times\left\{1+\frac{1}{4} \delta_{1}^{2}\left(\lambda(\sigma)^{-1}+\lambda\left(\sigma^{\prime}\right)^{-1}\right)^{-2}\left|s-s^{\prime}\right|^{2}\right\}^{-m / 2} \leqq h_{2}\left(t, t^{\prime}\right)^{2} .
\end{aligned}
$$

Therefore (63) and (66) imply the desired estimate (52). By a similar argument we have the estimate (53). This completes the proof of the part 1).

2) By an argument similar to the proof of the part 1) we know that the statement 2) is valid.

Proposition 3. 1) Let $m$ be an arbitrary integer such that $m>2 n$. Then we have the estimate

$$
\int_{R^{2 n}} h\left(t, t^{\prime}\right) d t \leqq C_{m}|a|_{0, m}
$$

2) Let $m$ be an arbitrary integer such that $m>2 n /(1-\varepsilon)$. Then we have the estimate

$$
\int_{R^{2 n}} k\left(t, t^{\prime}\right) d t \leqq C_{m}|a|_{0, m}
$$

Proof. 1) We note from (iii) in Corollary to Lemma 2 that $C_{2}^{-1} \leqq \lambda\left(\sigma^{\prime}\right) / \lambda(\sigma)$ $\leqq C_{2}$ in the support of $h_{1}$ and $h_{2}$.

We first prove estimate (67) for $h_{1}$. Since the characteristic function $\chi_{r_{1}}(\sigma)$ is a monotone non-increasing function of $|\sigma|$, we dominate $h_{1}\left(t, t^{\prime}\right)$ by

Then

$$
C|a|_{0,0} \chi_{r_{1}}\left(\frac{\sigma-\sigma^{\prime}}{\left(C_{2}+1\right) \lambda\left(\sigma^{\prime}\right)}\right) \chi_{r_{1}}\left(\frac{s-s^{\prime}}{2\left(C_{2}+2\right) \lambda\left(\sigma^{\prime}\right)^{-1}}\right) \text {. }
$$

$$
\int_{R^{2 n}} h_{1}\left(t, t^{\prime}\right) d t \leqq C_{0, n, r_{1}}|a|_{0,0} \lambda\left(\sigma^{\prime}\right)^{n} \lambda\left(\sigma^{\prime}\right)^{-n} \leqq C_{0, n, r_{1}}|a|_{0,0} .
$$

Next we prove (67) for $h_{2}$. We bound $h_{2}\left(t, t^{\prime}\right)$ from above:

$$
\begin{aligned}
h_{2}\left(t, t^{\prime}\right) \leqq & C_{m}|a|_{0, m} \chi_{r_{1}}\left(\frac{\sigma-\sigma^{\prime}}{\left(C_{2}+1\right) \lambda\left(\sigma^{\prime}\right)}\right) \\
& \times\left\{1+\frac{1}{4} \delta_{1}^{2}\left(1+C_{2}^{-1}\right)^{-2} \lambda\left(\sigma^{\prime}\right)^{2}\left|s-s^{\prime}\right|^{2}\right\}^{-m / 4}
\end{aligned}
$$

Therefore we have

$$
\begin{aligned}
\int_{R^{2 n}} h_{2}\left(t, t^{\prime}\right) d t \leqq C_{m}|a|_{0, m} \int_{R^{n}} \chi_{r_{1}}\left(\frac{\sigma-\sigma^{\prime}}{\left(C_{2}+1\right) \lambda\left(\sigma^{\prime}\right)}\right) d \sigma \\
\quad \times \int_{R^{n}}\left\{1+\frac{1}{4} \delta_{1}^{2}\left(1+C_{2}^{-1}\right)^{-2} \lambda\left(\sigma^{\prime}\right)^{2}\left|s-s^{\prime}\right|^{2}\right\}^{-m / 4} d s \\
\leqq C_{m}|a|_{0, m} \delta_{1}^{-n} \int_{R^{n}}\left(1+|s|^{2}\right)^{-m / 4} d s
\end{aligned}
$$


which is finite, independent of $t^{\prime}$ if $m>2 n$.

Thus (67) is proved for $h=h_{1}+h_{2}$.

2) We know from (iii) in Corollary to Lemma 2 that $C_{2}^{-1} \leqq \lambda\left(\sigma^{\prime}\right) / \lambda(\sigma) \leqq C_{2}$ in the support of $k_{1}$. A similar argument shows that

$$
k_{1}\left(t, t^{\prime}\right) \leqq C_{0}|a|_{0,0} \chi_{r_{1}}\left(\frac{s-s^{\prime}}{\left(C_{2}+1\right) \lambda\left(\sigma^{\prime}\right)^{-1}}\right) \chi_{r_{1}}\left(\frac{\sigma-\sigma^{\prime}}{2\left(C_{2}+1\right) \lambda\left(\sigma^{\prime}\right)}\right) .
$$

Then we have

$$
\int_{R^{2 n}} k_{1}\left(t, t^{\prime}\right) d t \leqq C_{0, n, r_{1}}|a|_{0,0}
$$

The desired estimate (68) is proved for $k_{1}$.

Next we prove (68) for $k_{2}$. We integrate (55) first with respect to $s$ and then to $\sigma$, and we have

$$
\begin{aligned}
& \int_{R^{2 n}} k_{2}\left(t, t^{\prime}\right) d t \leqq C_{m}|a|_{0, m} \\
& \quad \times \int_{R^{n}}\left\{1+\frac{1}{4} \delta_{1}^{2}\left(\lambda(\sigma)+\lambda\left(\sigma^{\prime}\right)\right)^{-2}\left|\sigma-\sigma^{\prime}\right|^{2}\right\}^{-m / 4} d \sigma \int_{R^{n}} \chi_{r_{1}}\left(\frac{s-s^{\prime}}{\lambda(\sigma)^{-1}+\lambda\left(\sigma^{\prime}\right)^{-1}}\right) d s \\
& \leqq C_{m, n, r_{1}}|a|_{0, m} \int_{R^{n}} \frac{\left(\lambda(\sigma)^{-1}+\lambda\left(\sigma^{\prime}\right)^{-1}\right)^{n} d \sigma}{\left\{1+\frac{1}{4} \delta_{1}^{2}\left(\lambda(\sigma)+\lambda\left(\sigma^{\prime}\right)\right)^{-2}\left|\sigma-\sigma^{\prime}\right|^{2}\right\}^{m / 4}} .
\end{aligned}
$$

We make use of the following lemma to handle the estimate of the righthand side of (69).

LEMMA 6. Let $\lambda(\xi)$ be a werght function of type $\varepsilon(0 \leqq \varepsilon<1)$. Then for any positive number $N \geqq \varepsilon /(1-\varepsilon)$ there exists a constant $C_{N}$ such that

$$
\frac{\lambda(\xi)}{\lambda(\eta)} \leqq C_{N}\left(1+\frac{|\xi-\eta|^{2}}{\lambda(\xi)^{2}}\right)^{N / 2}
$$

for any $\xi$ and $\eta$ in $\boldsymbol{R}^{n}$.

Admitting Lemma 6 for the moment, we continue the proof of Proposition 3-2). We are searching for a bound for the right-hand side of (69). We divide the right-hand side of (69) into two parts :

$$
\begin{aligned}
& J_{1}=C_{m}|a|_{0, m} \int_{\lambda(\sigma) \leqq \lambda\left(\sigma^{\prime}\right)} \frac{\left(\lambda(\sigma)^{-1}+\lambda\left(\sigma^{\prime}\right)^{-1}\right)^{n} d \sigma}{\left\{1+\frac{1}{4} \delta_{1}^{2}\left(\lambda(\sigma)+\lambda\left(\sigma^{\prime}\right)\right)^{-2}\left|\sigma-\sigma^{\prime}\right|^{2}\right\}^{m / 4}} \\
& J_{2}=C_{m}|a|_{0, m} \int_{\lambda(\sigma) \geqq \lambda\left(\sigma^{\prime}\right)} \frac{\left(\lambda(\sigma)^{-1}+\lambda\left(\sigma^{\prime}\right)^{-1}\right)^{n} d \sigma}{\left\{1+\frac{1}{4} \delta_{1}^{2}\left(\lambda(\sigma)+\lambda\left(\sigma^{\prime}\right)\right)^{-2}\left|\sigma-\sigma^{\prime}\right|^{2}\right\}^{m / 4}} .
\end{aligned}
$$

First we work out $J_{1}$. 


$$
\int_{1} \leqq C_{m}|a|_{0, m} \min \left\{1, \frac{1}{16} \delta_{1}^{2}\right\}^{-m / 4} \int_{R^{n}} \frac{\lambda(\sigma)^{-n} d \sigma}{\left\{1+\lambda\left(\sigma^{\prime}\right)^{-2}\left|\sigma-\sigma^{\prime}\right|^{2}\right\}^{m / 4}} .
$$

We use the inequality (70) in Lemma 6. If we take $\xi=\sigma^{\prime}$ and $\eta=\sigma$, then we have

$$
\lambda(\sigma)^{-1} \leqq C_{N} \lambda\left(\sigma^{\prime}\right)^{-1}\left(1+\frac{\left|\sigma-\sigma^{\prime}\right|^{2}}{\lambda\left(\sigma^{\prime}\right)^{2}}\right)^{N / 2}, \quad N=\varepsilon /(1-\varepsilon) .
$$

Then

$$
\begin{aligned}
J_{1} & \leqq C_{m} \min \left\{1, \frac{1}{16} \delta_{1}^{2}\right\}^{-m / 4}|a|_{0} \quad \int_{R^{n}} \frac{\lambda\left(\sigma^{\prime}\right)^{-n} d \sigma}{\left(1+\lambda\left(\sigma^{\prime}\right)^{-2}\left|\sigma-\sigma^{\prime}\right|^{2}\right)^{(m / 4)-(n N / 2)}} \\
& \leqq C_{m} \min \left\{1, \frac{1}{16} \delta_{1}^{2}\right\}^{-m / 4}|a|_{0, m} \int_{R^{n}} \frac{d \sigma}{\left(1+|\sigma|^{2}\right)^{(m / 4)-(n N / 2)}} .
\end{aligned}
$$

If $m>2 n /(1-\varepsilon)$, then $m>2 n(1+N)$. Hence $(m / 2)-n N>n$. Therefore the righthand side of (71) is finite and independent of $\sigma^{\prime}$. Thus we have the estimate

$$
J_{1} \leqq C_{m}|a|_{0, m}
$$

Next we consider $\int_{2}$. Since we know from Lemma 6 that

$$
\frac{1}{\lambda\left(\sigma^{\prime}\right)} \leqq C_{N} \frac{1}{\lambda(\sigma)}\left(1+\frac{\left|\sigma-\sigma^{\prime}\right|^{2}}{\lambda(\sigma)^{2}}\right)^{N / 2},
$$

we obtain the estimate that

$$
1+\frac{\left|\sigma-\sigma^{\prime}\right|^{2}}{\lambda\left(\sigma^{\prime}\right)^{2}} \leqq C\left(1+\frac{\left|\sigma-\sigma^{\prime}\right|^{2}}{\lambda(\sigma)^{2}}\right)^{N+1} .
$$

Then

$$
\begin{aligned}
J_{2} & \leqq C_{m}|a|_{0, m} \min \left\{1, \frac{1}{16} \delta_{1}^{2}\right\}^{-m / 4} \int_{R^{n}} \frac{\lambda\left(\sigma^{\prime}\right)^{-n} d \sigma}{\left(1+\lambda(\sigma)^{-2}\left|\sigma-\sigma^{\prime}\right|^{2}\right)^{m / 4}} \\
& \leqq C_{m} \min \left\{1, \frac{1}{16} \delta_{1}^{2}\right\}^{-m / 4}|a|_{1, m} \int_{R^{n}} \frac{\lambda\left(\sigma^{\prime}\right)^{-n} d \sigma}{\left(1+\lambda\left(\sigma^{\prime}\right)^{-2}\left|\sigma-\sigma^{\prime}\right|^{2}\right)^{m /(4(N+1))}} \\
& \leqq C_{m} \min \left\{1, \frac{1}{16} \delta_{1}^{2}\right\}^{-m / 4}|a|_{0, m} \int_{R^{n}} \frac{d \sigma}{\left(1+|\sigma|^{2}\right)^{m /(4(N+1))},}
\end{aligned}
$$

which is finite and independent of $\sigma^{\prime}$ if $m>2 n(N+1)$. Thus,

$$
J_{2} \leqq C_{m}|a|_{0, m} .
$$

Hence we have the estimate

$$
\int_{R^{2 n}} k_{2}\left(t, t^{\prime}\right) d t \leqq J_{1}+J_{2} \leqq C_{m}|a|_{0, m} .
$$

We have proved Proposition 3, assuming Lemma 6.

Proof of Lemma 6 . We take the basic weight function $\lambda_{1}(\xi)$ such that $\lambda(\xi)$ $=\lambda_{1}(\xi)^{\varepsilon}$. If $|\xi-\eta| \leqq r_{0} \lambda_{1}(\xi)$, then we have, from Lemma 2 , the estimates 


$$
C^{-1} \leqq \lambda_{1}(\xi) / \lambda_{1}(\eta) \leqq C
$$

Thus it is clear that (70) is valid in this case.

If $|\xi-\eta| \geqq r_{0} \lambda_{1}(\xi)$, then

$$
\begin{aligned}
\left(1+\frac{|\xi-\eta|^{2}}{\lambda(\xi)^{2}}\right)^{\varepsilon / 2(1-\varepsilon)} & \geqq\left(1+r_{0}^{2} \lambda_{1}(\xi)^{2(1-\varepsilon)}\right)^{\varepsilon / 2(1-\varepsilon)} \\
& \geqq r_{0}^{\varepsilon /(1-\varepsilon)} \lambda_{1}(\xi)^{\varepsilon} \geqq r_{0}^{\varepsilon /(1-\varepsilon)} \lambda_{\varepsilon}(\xi) \geqq r_{0}^{\varepsilon /(1-\varepsilon)} \lambda_{\varepsilon}(\xi) / \lambda_{\varepsilon}(\eta) .
\end{aligned}
$$

Therefore in this case we also have the estimate (70). This completes the proof of Lemma 6.

Now we have established Propositions 2 and 3 to apply the following lemma formulated by Calderón-Vaillancourt ([5]).

LEMMA 7. Let $t \rightarrow A_{t}$ be a continuous function from $\boldsymbol{R}^{n}$ to bounded operators on Hilbert space, and suppose that

$$
\begin{gathered}
\left\|A_{t}\right\| \leqq C, \\
\left\|A_{t} A_{t^{\prime}}^{*}\right\|^{1 / 2} \leqq h\left(t, t^{\prime}\right), \quad\left\|A_{t}^{*} A_{t^{\prime}}\right\|^{1 / 2} \leqq k\left(t, t^{\prime}\right),
\end{gathered}
$$

where $h\left(t, t^{\prime}\right)$ and $k\left(t, t^{\prime}\right)$ satisfy the estimates

$$
\sup _{t^{\prime}} \int_{\boldsymbol{R}^{n}} h\left(t, t^{\prime}\right) d t \leqq M, \quad \sup _{t^{\prime}} \int_{\boldsymbol{R}^{n}} k\left(t, t^{\prime}\right) d t \leqq M .
$$

Then for any compact set $K$ in $\boldsymbol{R}^{n}$ we have the estrmate

$$
\left\|\int_{K} A_{t} d t\right\| \leqq M
$$

where the constant $M$ is independent of $K$.

Proof. If $A=\int_{K} A_{t} d t$, we have $\|A\|^{2}=\left\|A^{*} A\right\|$ and more generally, by the spectral theorem, $\|A\|^{2 m}=\left\|\left(A^{*} A\right)^{m}\right\|$. We expand in an integral and use the fact that

$$
\begin{aligned}
& \left\|A_{1}^{*} A_{2} \cdots A_{2 m-1}^{*} A_{2 m}\right\| \\
& \leqq \min \left\{\left\|A_{1}^{*} A_{2}\right\| \cdots\left\|A_{2 m-1}^{*} A_{2 m}\right\|,\left\|A_{1}^{*}\right\|\left\|A_{2} A_{3}^{*}\right\| \cdots\left\|A_{2 m-1} A_{2 m-1}^{*}\right\|\left\|A_{2 m}\right\|\right\} .
\end{aligned}
$$

Taking the geometric mean of the two estimates and noting that $\left\|A_{t}\right\| \leqq C$ by hypothesis, we obtain

$$
\begin{gathered}
\|A\|^{2 m}=\left\|\left(A^{*} A\right)^{m}\right\| \leqq \iint \cdots \int\left\|A_{t_{1}}^{*}\right\|^{1 / 2}\left\|A_{t_{1}}^{*} A_{t_{2}}\right\|^{1 / 2} \cdots\left\|A_{t_{2 m-2}} A_{t_{2 m-1}}^{*}\right\|^{1 / 2} \\
\times\left\|A_{t_{2 m-1}}^{*} A_{t_{2 m}}\right\|^{1 / 2}\left\|A_{t_{2 m}}\right\|^{1 / 2} d t_{1} d t_{2} \cdots d t_{2 m} \\
\leqq C \iint \cdots \int k\left(t_{1}, t_{2}\right) h\left(t_{2}, t_{3}\right) \cdots h\left(t_{2 m-2}, t_{2 m-1}\right) \\
\times k\left(t_{2 m-1}, t_{2 m}\right) d t_{1} d t_{2} \cdots d t_{2 m}
\end{gathered}
$$




$$
\leqq C|K| M^{2 m-1},
$$

where $|K|$ is the volume of $K$. Hence,

$$
\|A\| \leqq(C|K| / M)^{1 / 2 m} M,
$$

and letting $m \rightarrow \infty$ we obtain $\|A\| \leqq M$.

Proposition 4. Let $\lambda(\xi)$ be a weight function of type $\varepsilon(0 \leqq \varepsilon<1)$. Suppose that $a(x, \xi)$ is a symbol function in $S^{0}(\lambda)$ and $S(x, \xi)$ is a phase function. If $a(x, \xi)$ has compact support, then the Fourier integral operator $A$ is $L^{2}$ bounded and has the following estimate

$$
\|A f\| \leqq C_{m}|a|_{0, m}\|f\|,
$$

where $m$ is an integer such that $m>2 n /(1-\varepsilon)$ and the constant $C_{m}$ is independent of the support of $a(x, \xi)$.

Proof. From the inequality $1^{\circ}$ ) and the estimates in Propositions 2 and 3 we know that $A_{t}$ defined in (22) satisfies the conditions of Lemma 7 if $a(x, \xi)$ has compact support. Applying Lemma 7 we have the conclusion of Proposition 4.

Now it remains to prove Theorem 1 when a symbol function $a(x, \xi)$ has non compact support. To handle this case we make use of the following lemma.

LEMMA 8. If $a(x, \xi)$ in $S^{0}(\lambda)$, then we have the estimate

$$
\|A f\| \leqq C_{m}|a|_{0, m} \sum_{|\alpha| \leqq m}\left\|\langle\xi\rangle^{m+|\alpha| \varepsilon} \partial_{\xi}^{\alpha}\left(e^{i S(0, \xi)} f(\xi)\right)\right\|
$$

for any function $f$ in $\mathcal{S}\left(R^{n}\right)$, where $m$ is an integer such that $m>n /(1-\varepsilon)$.

Admitting Lemma 8 for the moment, we prove Theorem 1.

Proof of Theorem 1. Let $a_{j}(x, \xi)$ be a bounded sequence in $S^{0}(\lambda)$ which converges to a symbol $a(x, \xi)$ in the topology of $S^{0}(\lambda)$. And suppose that each $a_{j}(x, \xi)$ has compact support. Then for $f$ in $\mathcal{S}\left(R^{n}\right)$

$$
\begin{aligned}
& \|A f\| \leqq \liminf _{j \rightarrow \infty}\left\|\left(A-A_{j}\right) f\right\|+\liminf _{j \rightarrow \infty}\|A, f\| \\
\leqq & \liminf _{j \rightarrow \infty}\left|a-a_{j}\right|_{0}, m \sum_{|\alpha| \leqq m}\left\|\langle\xi\rangle^{m+|\alpha| \varepsilon} \partial_{\xi}^{\alpha}\left(e^{i S(0, \xi)} f(\xi)\right)\right\|+\liminf _{j \rightarrow \infty} C_{m}\left|a_{j}\right|_{0, m}\|f\| \\
\leqq & C_{m}|a|_{0}, m\|f\| .
\end{aligned}
$$

We have proved the proof of Theorem 1, assuming Lemma 8.

Proof of Lemma 8. Set

$$
B g(x)=\int_{\boldsymbol{R}^{n}} e^{\imath(S(x, \xi)-S(0, \xi))} a(x, \xi) g(\xi) d \xi .
$$


Then $A f(x)=B\left(e^{i S(0, \xi)} f(\xi)\right)$. Hence if suffices to prove that the estimate

$$
\|B g\| \leqq C_{m}|a|_{0, m} \sum_{|\alpha| \leqq m}\left\|\langle\xi\rangle^{m+|\alpha| \varepsilon} \partial_{\xi}^{\alpha} g(\xi)\right\|
$$

holds.

Let $\varphi_{(s, \sigma)}(x, \xi)$ be a partition of unity in Lemma 3. For $\sigma^{\prime}$ in $\boldsymbol{R}^{n}$ we set

$$
\varphi_{\sigma^{\prime}}(\xi)=\int_{R^{n}} \varphi_{\left(s, \sigma^{\prime}\right)}(0, \xi) d s .
$$

Then

$$
\left|\partial_{\xi}^{\beta} \varphi_{\sigma^{\prime}}(\xi)\right| \leqq C_{\beta} \lambda(\xi)^{-1 \beta \mid} \chi_{r_{1}}\left(\lambda\left(\sigma^{\prime}\right)^{-1}\left(\xi-\sigma^{\prime}\right)\right) .
$$

Define

$$
B_{\left(s, \sigma, \sigma^{\prime}\right)} g(x)=\int_{R^{n}} e^{\iota(S(x, \xi)-S(0, \xi))} a_{(s, \sigma)}(x, \xi) \varphi_{\sigma^{\prime}}(\xi) g(\xi) d \xi .
$$

Then we have

$$
B g(x)=\int_{R^{n}} B\left(s, \sigma, \sigma^{\prime}\right) g(x) d s d \sigma d \sigma^{\prime} .
$$

Here we note that $(75)$ is of the form similar to $H_{\left(s, \sigma, 0, \sigma^{\prime}\right)}(x, 0)$.

Let $L_{0}$ be a partial differential operator of order 1 :

where

$$
L_{0}=\rho_{0}^{-2}\left\{1-i\left(\lambda(\sigma)^{-1}+\lambda\left(\sigma^{\prime}\right)^{-1}\right)^{-2} \nabla_{\xi}(S(x, \xi)-S(0, \xi)) \cdot \nabla_{\xi}\right\},
$$

$$
\rho_{0}=\left\{1+\left(\lambda(\sigma)^{-1}+\lambda\left(\sigma^{\prime}\right)^{-1}\right)^{-2}\left|\nabla_{\xi}(S(x, \xi)-S(0, \xi))\right|^{2}\right\}^{1 / 2} .
$$

Then integration by parts in (75) yields that

$$
B_{\left(s, \sigma, \sigma^{\prime}\right)} g(x)=\int_{R^{n}} e^{\imath(S(x, \xi)-S(0, \xi))\left({ }^{t} L_{0}\right)^{m}\left(a_{(s, \sigma)}(x, \xi) \varphi_{\sigma^{\prime}}(\xi) g(\xi)\right) d \xi .}
$$

By a similar argument in the proof of Proposition 1 we obtain the estimate

$$
\begin{aligned}
\left|B_{\left(s, \sigma, \sigma^{\prime}\right)} g(x)\right| \leqq C_{m}|a|_{0, m} \chi_{r_{1}}(\lambda(\sigma)(x-s)) \\
\quad \times \int_{R^{n}} \frac{\chi_{r_{1}}\left(\lambda(\sigma)^{-1}(\xi-\sigma)\right) \chi_{r_{1}}\left(\lambda\left(\sigma^{\prime}\right)^{-1}\left(\xi-\sigma^{\prime}\right)\right)}{\left\{1+\delta_{1}^{2}\left(\lambda(\sigma)^{-1}+\lambda\left(\sigma^{\prime}\right)^{-1}\right)^{-2}|x|^{2}\right\}^{m / 2}} \\
\quad \times \sum_{|\beta| \leqslant m} \lambda\left(\sigma^{\prime}\right)^{|\beta|} \partial_{\xi}^{\beta}\left(\varphi_{\sigma^{\prime}}(\xi) g(\xi)\right) \mid d \xi .
\end{aligned}
$$

We set

and

$$
\begin{aligned}
\tilde{H}_{\left(s, \sigma, \sigma^{\prime}\right)}( & x, \xi)=C_{m}|a|_{0, m} \chi_{r_{1}}(\lambda(\sigma)(x-s)) \\
& \times \frac{\chi_{r_{1}}\left(\lambda(\sigma)^{-1}(\xi-\sigma)\right) \chi_{r_{1}}\left(\lambda\left(\sigma^{\prime}\right)^{-1}\left(\xi-\sigma^{\prime}\right)\right)}{\left\{1+\delta_{1}^{2}\left(\lambda(\sigma)^{-1}+\lambda\left(\sigma^{\prime}\right)^{-1}\right)^{-2}|x|^{2}\right\}^{m / 2}}
\end{aligned}
$$

$$
G_{\sigma^{\prime}}(\xi)=\sum_{\mid \beta \backslash \leqq m} \lambda\left(\sigma^{\prime}\right)^{|\beta|}\left|\partial_{\xi}^{\beta}\left(\varphi_{\sigma^{\prime}}(\xi) g(\xi)\right)\right| \text {. }
$$


Then

$$
\left|B_{\left(s, \sigma, \sigma^{\prime}\right)} g(x)\right| \leqq \int_{R^{n}} \tilde{H}_{\left(s, \sigma, \sigma^{\prime}\right)}(x, \xi) G_{\sigma^{\prime}}(\xi) d \xi .
$$

By a similar argument in the proof of Propositions 2 and 3 there exists a positive function $\tilde{h}\left(s, \sigma, \sigma^{\prime}\right)$ such that

$$
\begin{aligned}
& \int_{R^{n}} \widetilde{H}_{\left(s, \sigma, \sigma^{\prime}\right)}(x, \xi) d x \leqq C_{m}|a|_{0, m} \tilde{h}\left(s, \sigma, \sigma^{\prime}\right), \\
& \int_{R^{n}} \widetilde{H}_{\left(s, \sigma, \sigma^{\prime}\right)}(x, \xi) d \xi \leqq C_{m}|a|_{0, m} \tilde{h}\left(s, \sigma, \sigma^{\prime}\right)
\end{aligned}
$$

and

$$
\int_{R^{2 n}} \tilde{h}\left(s, \sigma, \sigma^{\prime}\right) d s d \sigma \leqq C,
$$

where $m$ is an arbitrary integer such that $m>n /(1-\varepsilon)$. By Schur's lemma and (77). (78) and (79) we have

$$
\left\|B_{\left(s, \sigma, \sigma^{\prime}\right)} g\right\| \leqq C_{m}|a|_{0, m} \tilde{h}\left(s, \sigma, \sigma^{\prime}\right)\left\|G_{\sigma^{\prime}}(\sigma)\right\| .
$$

Then by Minkowski's inequality and (80) we have

$$
\begin{aligned}
\|B g\| & \leqq \int_{R^{3 n}}\left\|B_{\left(s, \sigma, \sigma^{\prime}\right)} g\right\| d s d \sigma d \sigma^{\prime} \\
& \leqq C_{m}|a|_{0, m} \int_{R^{3 n}} \tilde{h}\left(s, \sigma, \sigma^{\prime}\right)\left\|G_{\sigma^{\prime}}(\xi)\right\| d s d \sigma d \sigma^{\prime} \\
& \leqq C_{m}|a|_{0, m} \int_{R^{n}}\left\|G_{\sigma^{\prime}}(\xi)\right\| d \sigma^{\prime} .
\end{aligned}
$$

If $\left|\xi-\sigma^{\prime}\right| \leqq r_{1} \lambda\left(\sigma^{\prime}\right)$, then Corollary to Lemma 2 implies

$$
C_{2}^{-1} \leqq \lambda(\xi) / \lambda\left(\sigma^{\prime}\right) \leqq C_{2} .
$$

Hence we have the estimate

$$
C^{-1} \leqq \frac{1+\lambda(\xi)^{-2}|\xi|^{2}}{1+\lambda\left(\sigma^{\prime}\right)^{-2}\left|\sigma^{\prime}\right|^{2}} \leqq C
$$

on the support of $G_{\sigma^{\prime}}(\xi)$. Then

$$
\begin{aligned}
& \int_{R^{n}}\left\|G_{\sigma^{\prime}}(\xi)\right\| d \sigma^{\prime} \leqq C_{m} \int_{R^{n}}\left(1+\lambda\left(\sigma^{\prime}\right)^{-2}\left|\sigma^{\prime}\right|^{2}\right)^{-m / 2} \\
& \times\left\|\left(1+\lambda(\xi)^{-2}|\xi|^{2}\right)^{m / 2} G_{\sigma^{\prime}}\right\| d \sigma^{\prime} .
\end{aligned}
$$

By Leibniz's rule and (74) we obtain the estimate 


$$
\begin{aligned}
& \left\|\left(1+\lambda(\xi)^{-2}|\xi|^{2}\right)^{m / 2} G_{\sigma^{\prime}}(\xi)\right\| \\
\leqq & C_{m} \sum_{|\beta| \leqq m}\left\|\left(1+\lambda(\xi)^{-2}|\xi|^{2}\right)^{m / 2} \lambda(\xi)^{|\beta|}\left|\partial_{\xi}^{\beta}\left(\varphi_{\sigma^{\prime}}(\xi) g(\xi)\right)\right|\right\| \\
\leqq & C_{m} \sum_{|\beta| \leqq m} \sum_{\gamma+\delta=\beta}\left\|\left(1+\lambda(\xi)^{-2}|\xi|^{2}\right)^{m / 2} \lambda(\xi)^{|\beta|}\left|\partial_{\xi}^{\gamma} \varphi_{\sigma^{\prime}}(\xi)\right|\left|\partial_{\xi}^{\delta} g(\xi)\right|\right\| \\
\leqq & C_{m} \sum_{|\beta| \leqq m} \sum_{|\delta| \leqq|\beta|}\left\|\left(1+\lambda(\xi)^{-2}|\xi|^{2}\right)^{m / 2} \lambda(\xi)^{|\delta|}\left|\partial_{\xi}^{\delta} g(\xi)\right|\right\| \\
\leqq & C_{m} \sum_{|\alpha| \leqq m}\left\|\langle\xi\rangle^{m+s|\alpha|}\left|\partial_{\xi}^{\alpha} g(\xi)\right|\right\| .
\end{aligned}
$$

And from Lemma 6 we have the estimate

$$
\int_{R^{n}}\left(1+\lambda\left(\sigma^{\prime}\right)^{-2}\left|\sigma^{\prime}\right|^{2}\right)^{-m / 2} d \sigma^{\prime} \leqq C_{m, s} \int_{R^{n}}\left(1+\lambda(0)^{-2}\left|\sigma^{\prime}\right|^{2}\right)^{-(1-c) m / 2} d \sigma^{\prime},
$$

which is finite if $m>n /(1-\varepsilon)$. Hence from (81), (82) and (83) we have the estimate

$$
\int_{R^{n}}\left\|G_{\sigma^{\prime}}(\xi)\right\| d \sigma^{\prime} \leqq C_{m} \sum_{|\beta| \leqq m}\left\|\langle\xi\rangle^{m+\varepsilon|\beta|}\left|\partial_{\xi}^{\beta} g(\xi)\right|\right\|
$$

Thus,

$$
\|B g\| \leqq C_{m}|a|_{0, m} \sum_{|\beta| \leqq m}\left\|\langle\xi\rangle^{m+\varepsilon|\beta|}\left|\partial_{\xi}^{\beta} g(\xi)\right|\right\| .
$$

This completes the proof of Lemma 8 and the proof of Theorem 1 .

Acknowledgements. The author is indebted to Prof. D. Fujiwara for valu. able advice and to Prof. H. Komatsu for hearty encouragement.

\section{REFERENCES}

[1] Asada, K., AND Fujiwara, D., On the oscillatory integral transformations in $L^{2}\left(\boldsymbol{R}^{n}\right)$, Japan. J. Math., 4 (1978), 299-361.

[2] Beals, R. and Fefferman, C., Spatially inhomogeneous pseudodifferential operators, I, Comm. Pure Appl. Math., 27 (1974), 1-24.

[3] Beals, R., A general calculus of pseudodifferential operators, Duke Math. J., 42 (1975), 1-42.

[4] Boutet de Monvel, L., Hypoelliptic operators with double characteristics and related pseudodifferential operators, Comm. Pure Appl. Math., 27 (1974), 585-639.

[5] Calderôn, A.P. ANd Vaillancourt, R., A class of bounded pseudo-differential operators, Proc. Nat. Acad. Sci. USA, 69 (1972), 1185-1187.

[6] Fujiwara, D., On the boundedness of integral transformations with highly oscilla. tory kernels, Proc. Japan Acad., 51 (1975), 96-99.

[7] Fujiwara, D., A global version of Eskin's theorem, J. Fac. Sci. Univ Tokyo, Sec. IA, 24 (1977), 327-340.

[8] Hörmander, L., Fourier integral operators, I, Acta Math., 127 (1971), 79-183.

[9] Hörmander, L., The Weyl calculus of pseudo-differential operators, Comm. Pure Appl. Math., 32 (1979), 359-443. 
[10] Kumano-go, H., Pseudo-differential operators, Iwanami, Tokyo, Japan, (1974), (Japanese).

[11] Kumano-go, H. and Taniguchi, K., Oscillatory integrals of symbols of pseudodifferential operators on $\boldsymbol{R}^{n}$ and operators of Fredholm type, Proc. Japan Acad., 49 (1973), 397-402.

[12] Kumono-Go, H., A caluculus of Fourier integral operators on $R^{n}$ and the fundamental solution for an operator of hyperbolic type, Comm. in P.D.E., 1 (1976), $1-44$.

[13] AsAda, K., On the $L^{2}$ boundedness of Fourier integral operators in $\boldsymbol{R}^{n}$, Proc. Japan Acad., 57 (1981), 249-253.

Department of Elementary Education

Chiba Keizal College

4-3-30, TODOROKI-CHO

ChIBA-shi, 260, Japan 